\title{
Computational Reduction of Specimen Noise to Enable Improved Thermography Characterization of Flaws in Graphite Polymer Composites
}

\author{
William P. Winfree*a, Patricia A. Howell ${ }^{\mathrm{b}}$ and Joseph N. Zalameda ${ }^{\mathrm{b}}$ \\ ${ }^{a}$ NASA Langley Research Center, Research Directorate, MS 225, Hampton, VA 23681; ${ }^{\text {NAASA }}$ \\ Langley Research Center, Nondestructive Evaluation Sciences Branch MS 231, Hampton, VA \\ 23681
}

\begin{abstract}
Flaw detection and characterization with thermographic techniques in graphite polymer composites are often limited by localized variations in the thermographic response. Variations in properties such as acceptable porosity, fiber volume content and surface polymer thickness result in variations in the thermal response that in general cause significant variations in the initial thermal response. These result in a "noise" floor that increases the difficulty of detecting and characterizing deeper flaws. A method is presented for computationally removing a significant amount of the "noise" from near surface porosity by diffusing the early time response, then subtracting it from subsequent responses. Simulations of the thermal response of a composite are utilized in defining the limitations of the technique. This method for reducing the data is shown to give considerable improvement characterizing both the size and depth of damage. Examples are shown for data acquired on specimens with fabricated delaminations and impact damage.
\end{abstract}

Keywords: Nondestructive Evaluation, Thermography, Infrared Inspections, Composites

\section{INTRODUCTION}

Thermography has been shown to be an effective method of rapid inspection of large carbon fiber reinforced polymer (CFRP) composite structures. Increased utilization of composites in commercial and military aircraft increases the need for viable inspection techniques for ensuring aircraft safety and reliability. Thermography has been shown to have great potential for detection of delaminations in CFRP composites [1-5]. Efforts have included a variety of heating and data reduction techniques to improve the detectability and assessment of the size and depth of delaminations. Thermography is particularly effective in cases where the delamination is close to the inspected surface in a single sided inspection or if an inspection can be performed where one side of the specimen is heated and the in thermal response is measured from the opposite side.

Single sided inspections of composites have some difficulty detecting delaminations which are deeper than one half of the thickness of the composite. This is not an inherent limitation of the thermographic technique, since it is possible to detect 5\% material loss in aluminum plates. For thermographic inspection, there are two significant differences between detecting material loss in metals and detecting delaminations in composites. The first is material loss which is similar to flat bottom holes and does not have the thin air gap. The air gap has a thermal contact resistance that allows heat to cross from the upper surface to the lower surface, therefore the thermal contrast over the delamination is not as large as material loss. A second factor that increases the difficulty of detecting delaminations in composites is the inherent inhomogeneities in the composites such as small spatial variations in fiber volume content or porosity. These inhomogeneities cause spatial variation in the thermal response of the composites, causing a higher "noise" floor in the measurement that is not present for a homogeneous metal plate.

*william.p.winfree@nasa.gov; phone 1-757-864-4963 
If one assumes the these small inhomogeneities have a thermal response similar to inhomogeneities in surface heating, then is it possible to estimate the "noise" contribution of future data based on early time response. The predicted later time response, based only on the early time response, can be subtracted from later time responses to improve the detectability of deeper flaws.
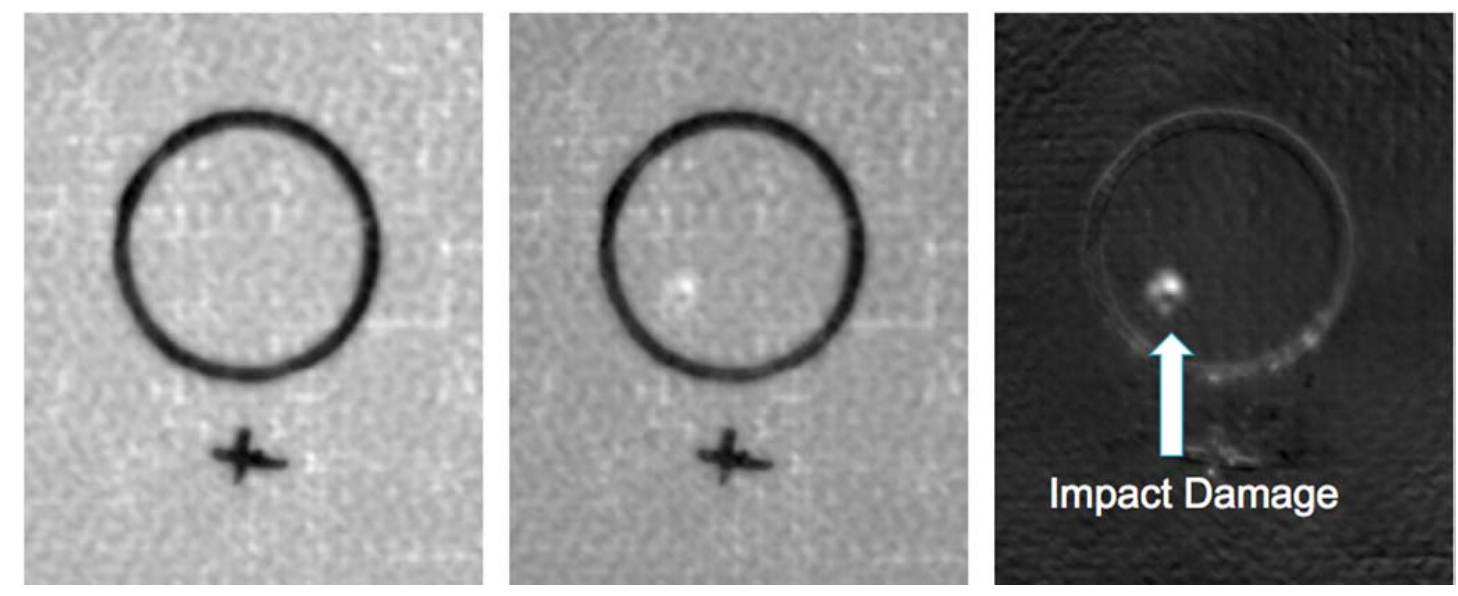

Figure 1. The left and center thermographic images are acquired on a composite specimen prior to and post impact respectively. The image on the far right is the difference between the two images on it's left after they have been aligned.

An example of the "material noise" reducing the detectability of subsurface damage is shown in Fig 1. The figure has two images of the thermal response of a composite, the one on the left acquired before an impact event, the one in the center acquired after an impact event. A comparison of the two images shows there is evidence of impact damage, however the magnitude of the thermal response of the impact damage is on the order of the magnitude of the "material noise". If one aligns these two images and calculates the difference between the two aligned images, the image on the far right is obtained. The impact damage is obvious in the difference image, after the "material noise" has been computationally removed.

The thermal response of the initial state of a specimen is not always available for comparison to data that is acquired after a suspected damage inducing event. This paper describes a two-dimension finite difference method for estimating the late time thermal "material noise" that results from near surface inhomogeneities or surface heating from the early time response, then removing those "noise sources" from thermal response acquired at later times. A simple discussion of the technique is given in section 2. The method is applied to a simulation of the thermal response of a near surface void, to demonstrate the two dimension finite element technique gives a reasonable approximation to the thermal response at later times in section 3. Section 4 gives a brief description of thermographic measurement system. The application of the technique to data acquired on composite specimens is discussed in section 5. The capabilities and limitation of the technique are discussed in section 6 .

\section{METHOD OF REDUCTION OF MATERIAL NOISE}

The equation for heat flow in a homogeneous material is given by

$$
\alpha \nabla T(x, y, z, t)=\frac{\partial T(x, y, z, t)}{\partial t}
$$

where $\alpha$ is the material thermal diffusivity. Assuming the an inhomogeneity in (1) makes only a small perturbation to the temperature flow in the $\mathrm{z}$ direction of the material, then to a good approximation the thermal response is given by

$$
T(x, y, z, t)=T_{x y}(x, y, t) T_{z}(z, t)
$$

with the equations for the two components given by

$$
\alpha \nabla T_{Z}(z, t)=\frac{\partial T_{Z}(z, t)}{\partial t}
$$


and

$$
\alpha \nabla T_{x y}(x, y, t)=\frac{\partial T_{x y}(x, y, t)}{\partial t}
$$

The evolution of the thermal response of the material noise is calculated from a numerical solution of equation 1, using an initial condition of $T_{x y}\left(x, y, t_{0}\right)$ which is the measured thermal response at some relatively early time (typically the first image where the imager is not saturated after flash). The surface temperature is evolved with the finite difference equation

$$
T_{m, n}^{i+1}=(1-r) T_{m, n}^{i}+\frac{r}{4}\left(T_{m-1, n}^{i}+T_{m+1, n}^{i}+T_{m, n-1}^{i}+T_{m, n+1}^{i}\right)
$$

where $i$ is the time step, $m$ and $n$ are the indexes of the pixel of interest in the thermal image and

$$
r=\frac{4 \tau \alpha}{h^{2}}
$$

where $\tau$ is the finite difference time step and $h$ is the pixel spacing in the thermal response. To ensure a solution stability for the estimated thermal response, $r$ is set to $0.5[6]$.

To predict future thermal responses of the small near surface material variations from initial responses, equation 5 is applied to transform an initial image. Multiple time steps are typically calculated by an algorithm implementing equation 5 to create a time series of images. A region of the image, where it is assumed that there is no flaw, is compared to the same region on the calculated time series to find the best match based on a least squared linear fit pixel by pixel. The member of the time series of the calculated response that best matches the measured response in that region is used to correct the entire measured response.

\section{APPLICATION OF METHOD TO SIMULATION RESULTS}

To illustrate that the method could estimate the thermal response of a small subsurface flaw, a 2D finite element routine was used to estimate the thermal response for a small near surface void in a homogeneous block. The void was $0.01 \mathrm{~cm}$ x $0.01 \mathrm{~cm}$ air filled and placed $0.01 \mathrm{~cm}$ below surface. The size of the block was $1 \mathrm{~cm}$ wide and $0.3 \mathrm{~cm}$ thick. A simulated $0.01 \mathrm{sec}$ impulse heat flux was applied at surface. The material thermal diffusivity was $0.005 \mathrm{~cm}^{2} / \mathrm{sec}$. The simulated results indicated the heat diffuses around the void and does not significantly perturb the heat flow. Profiles of the thermal response across the void at select times are shown with dashed lines in Fig. 2. The nominal temporal temperature response has been subtracted to improve the visibility of the void. As is expected, the perturbation due to the void both decreases and spatially expands with time.

The temperature profiles from the two dimensional finite element simulation were used to estimate the surface temperature for a long thin void in a thin plate. In Fig. 2, the solid lines are the difference between finite element simulation profiles and the profiles estimated from using multiple iterations of equation 5 , using the temperature profile at 0.033 seconds as the initial temperature profile. As can be seen from the figure, the effect of the small void can be mostly removed using the estimated thermal response. The number of iterations required to find the best match at any time during the simulation is shown in figure 3. Ideally, there would be a linear relationship between the number of iterations required for the best match and the simulation time, however, since the in-plane and surface normal responses are not truly separable as is assumed in equation 2, it is reasonable that there is not a linear relationship in this figure. Also, shown in the figure is a quadratic fit of these points. Using the initial slope of this fit, it is possible from the spacing of the pixels and the known time steps to estimate the diffusivity from this plot using equation 6 . This results in an estimate of the in-plane diffusivity for the simulation of $0.0048 \mathrm{~cm}^{2} / \mathrm{sec}$ which is in good agreement with the 0.0049 $\mathrm{cm}^{2} / \mathrm{sec}$ input to the simulation. 


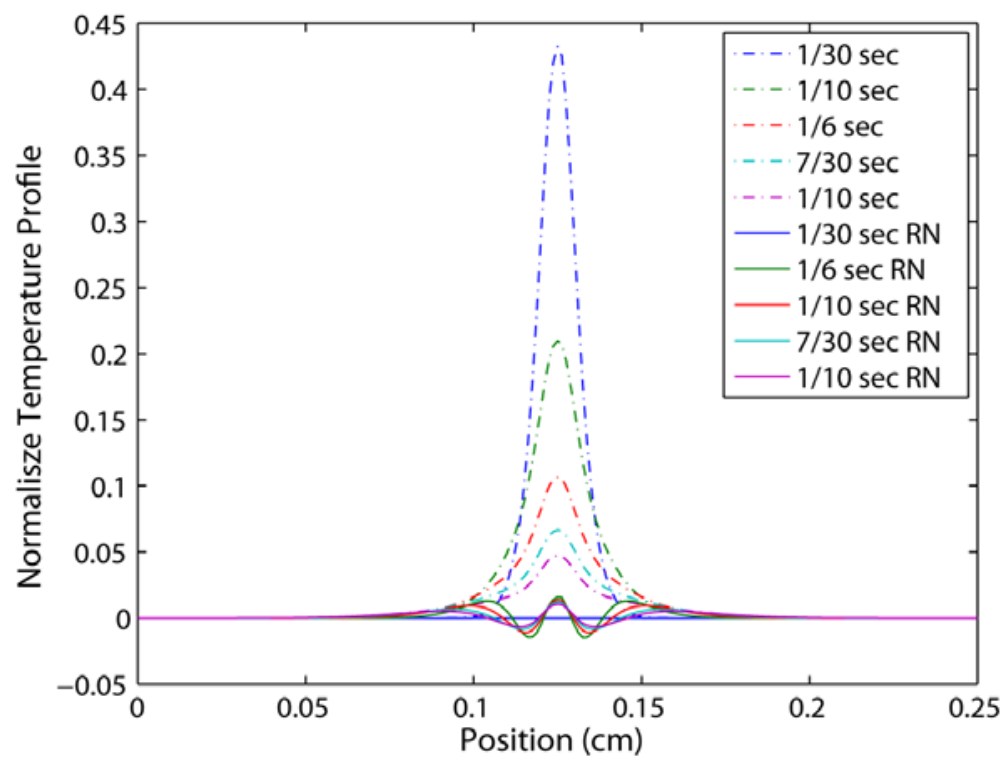

Figure 2. The thermal profiles as a function of time for a $0.01 \mathrm{~cm} \mathrm{x} 0.01 \mathrm{~cm}$ air filled void $0.01 \mathrm{~cm}$ below surface (dashed lines) and the same profiles minus the thermal profiles estimated from an early time response.

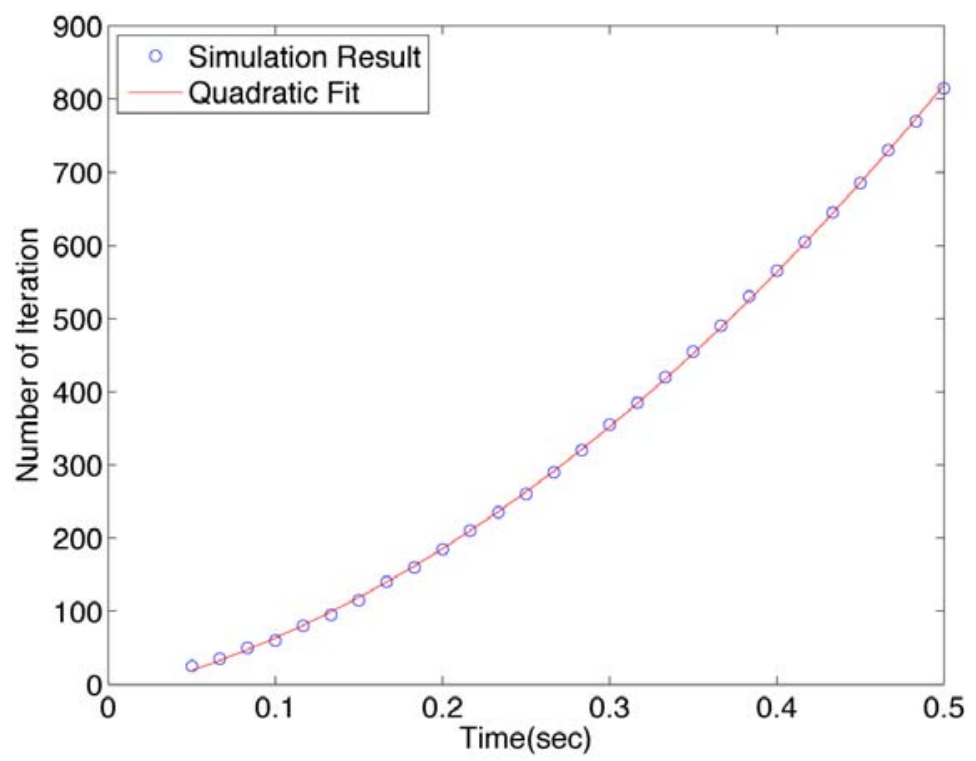

Figure 3. Number of iterations (with the initial condition of the surface temperature at 0.133 seconds) to obtain smallest summed squared difference between the diffused early time response at a later time.

\section{THERMOGRAPHIC MEASUREMENT SYSTEM}

A commercially available computer controlled flash thermography system was used to perform measurements on composite specimens. The system consists of an infrared (IR) imager with a 320 x 256 sensor array of IndiumAntimonide elements and two 4800-Joule xenon photographic flash tubes mounted in a hood to contain and focus the flash. The hood is $36.8 \mathrm{~cm}$ wide by $26.7 \mathrm{~cm}$ deep by $40.6 \mathrm{~cm}$ tall and is configured such that the IR camera views the inspection surface directly. The flash produces a flash energy density of approximately 7 joules per square centimeter at the mouth of the hood. The flash lamps provide a uniform illumination of within $10 \%$ across an area of $25 \mathrm{~cm} \times 33 \mathrm{~cm}$ of a flat reference standard at the mouth of the hood. 
Thermographic inspection was accomplished by placing the hood on the material to be inspected. For these measurements the bottom of the hood completely surrounded the specimens. The flash lamps are triggered by the computer that controls the data acquisition process. Thermographic images of the specimen are captured by the infrared camera for a predetermined amount of time and stored in the computer for further analysis. The camera's noise equivalent temperature difference $(\mathrm{NE} \Delta \mathrm{T})$, cited by the manufacturer, is $0.025^{\circ} \mathrm{C}$ operating the detector in the 3 to 5 micrometer wavelength range.

\section{NOISE REDUCTION IN DATA FROM COMPOSITE SPECIMENS}

Data was acquired on three different composite specimens to evaluate the capability of the technique for reducing the "material noise" in the specimen. The first specimen considered was a composite with impact damage. The impact damage is clearly detectable in the thermographic response. Of interest was obtaining an image of the damage at the earliest time possible, since the thermal profile of the delamination is closest to the size of the flaw at that time. Fig. 4 shows the comparison of two images of the thermal response, the upper image is not processed and the lower one has had the noise reduction procedure applied to the data. As can be seen from a comparison of the two images, the noise reduction routine significantly improves the visibility of the impact damage at early times. This is critical, since the best measurement of the size of the damage is at early times, before there is significant in-plane diffusion.
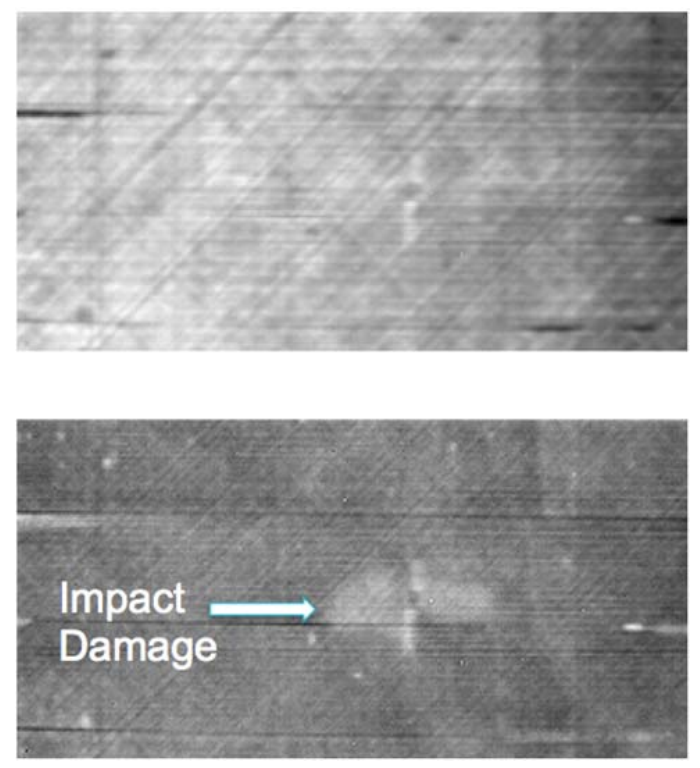

Figure 4. Comparison of the as acquire thermal response 0.5 seconds after flash heating of the an impacted composite (upper image) and the same data with the thermal response estimated for an extrapolation of the early time response subtracted(lower image).

Another visualization of the capabilities of the noise reduction technique is shown in Fig. 5. This figure shows a plot of multiple lines across the noise reduced and original images, in a region with no detectable impact damage. As can be seen from the figure, the noise reduction is about a factor of three. The time dependence of noise for a region with no detectable impact damage is shown in Fig. 6. The figure shows both the time dependence of the original data and the data where the noise reduction has been applied. For the original data, the noise decreases with time, which is expected if the primary source of the noise is small regions of near surface material of inhomenities in the composite, similar to what was seen in Fig 2., where the amplitude of the response over the small near surface void gets smaller with time. However, for all times within the window of interest, the noise reduction routine reduces the noise by a factor of approximately two or more. 


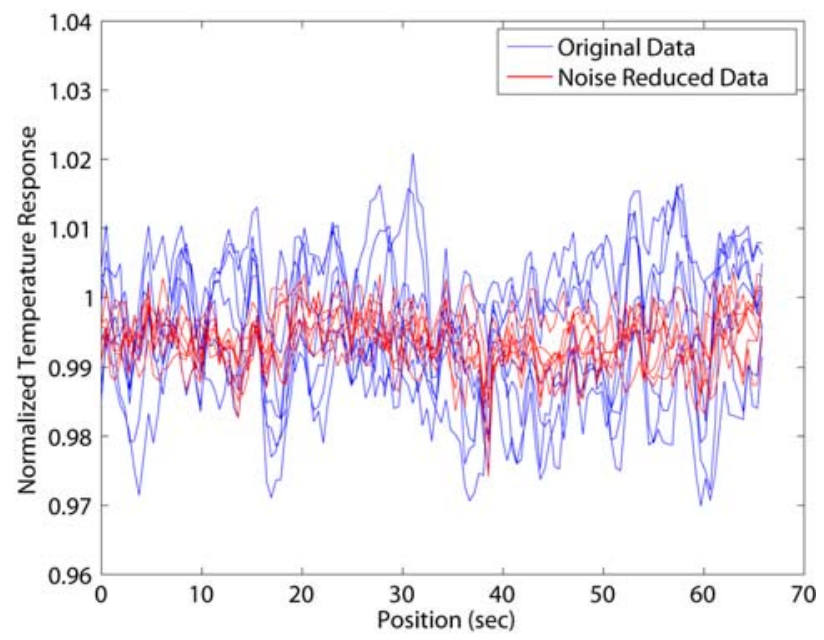

Figure 5. Comparison of data along horizontal lines in the thermal response at 0.133 seconds showing the reduction in noise resulting from subtracting the estimated thermal response from the initial thermal response.

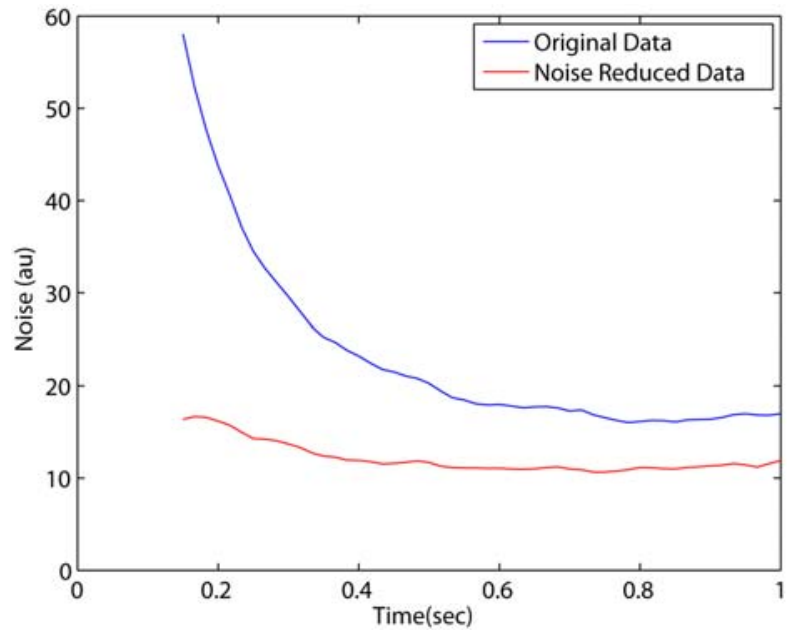

Figure 6. Comparison of time evolution of noise in original data and in data where the noise has been computationally reduced be estimating later thermal responses from an early thermal response.

The number of iterations required to find the best match at any time during the data set is shown in figure 7. Ideally, once again, there would be a linear relationship between the number of iterations required for the best match and the simulation time, however, since the in-plane and surface responses are not truly separable as is assumed in equation 2 , it is reasonable that there is not a linear relationship in this figure. Also, shown in the figure is a quadratic fit of these points. Using the initial slope of this fit, it is possible from the known spacing of the pixels and the known time steps to estimate the in-plane diffusivity from this plot using equation 6 , which results in an estimate of the in-plane diffusivity for the simulation of $0.0096 \mathrm{~cm}^{2} / \mathrm{sec}$ which is larger than the measured through the thickness diffusivity of 0.0049 $\mathrm{cm}^{2} / \mathrm{sec}$, as would be expected for the thermal diffusivity in the direction of the carbon fibers. 


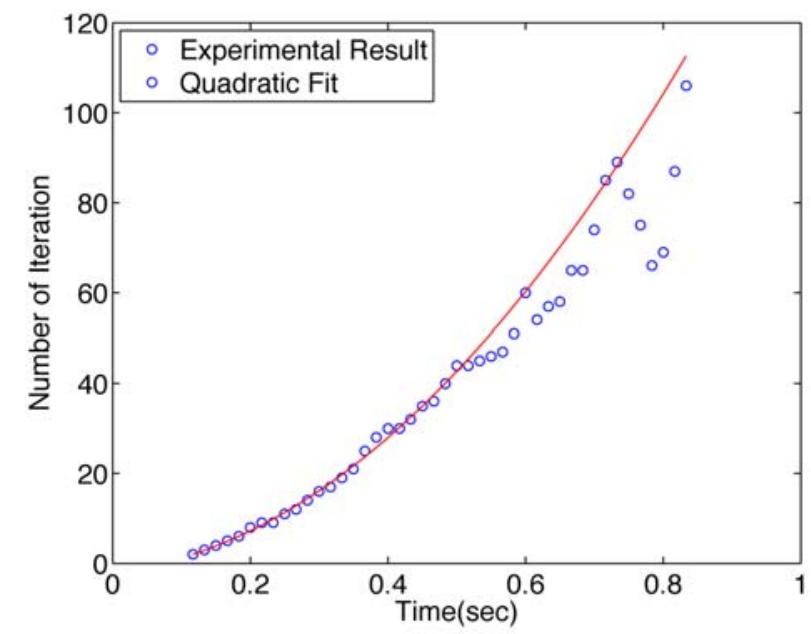

Figure 7. Number of iterations ( with initial condition of surface temperature at 0.0167 seconds) to obtain smallest summed squared difference between the diffused early time response at a later time for data from impacted composite.
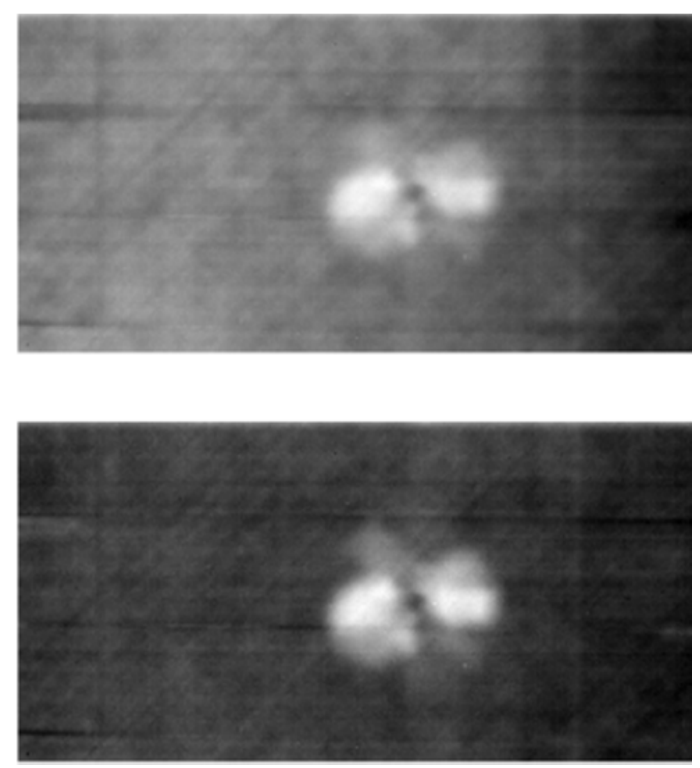

Figure 8. Comparison of the as-acquired thermal response at 0.5 seconds after flash heating of the an impacted composite (upper image) and the same data with the thermal response estimated for an extrapolation of the early time response subtracted(lower image)

The thermal response at a later time is shown in Fig. 8 for the same composite specimen as shown in Fig 4. The upper image is not processed and the lower one has had the noise reduction procedure applied to the data. The high frequency noise in the original data is not as significant at later times as it was for times close to the impulse heating. However, there is low frequency content that is perhaps a result of slight variations in the initial heating of the specimen. The noise reduction routine significantly removes this low frequency content and improves the visibility of the impact damage that is at a greater depth in the composite.

A second specimen used for testing the viability of the noise reduction technique was a composite specimen with delaminations in the back half relative to the measurement side. The 10 ply quasi-isotropic composite panel with a layup of $[0,45 /, 90,-45,0 /, 45,90,-45 /, 0,90 /]$ was $30.5 \times 30.5$ centimeters and 0.19 centimeters thick. The delamination 
defect areas were squares with sizes of 14.5, 6.54, 3.6, and 1.6 square centimeters. The defects were buried at depths of $50,60,70,80$, and 90 percent of the total thickness. A schematic of the defect layout is shown in Fig. 9.

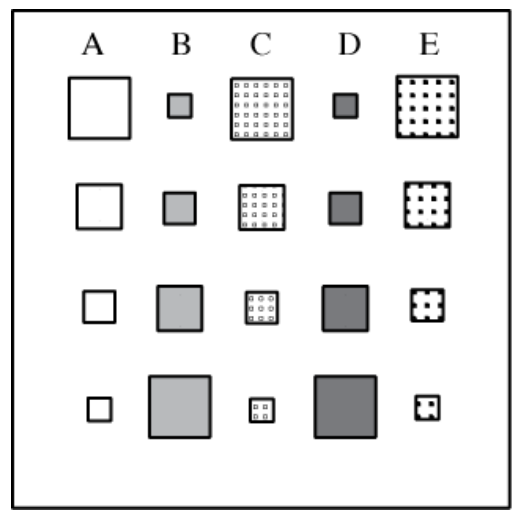

Delaminations

between

plies

A: $9-10(90 \%$

B: $8-9(80 \%)$

C: $7-8(70 \%)$

D: 6-7 $(60 \%)$

E: $5-6(50 \%)$

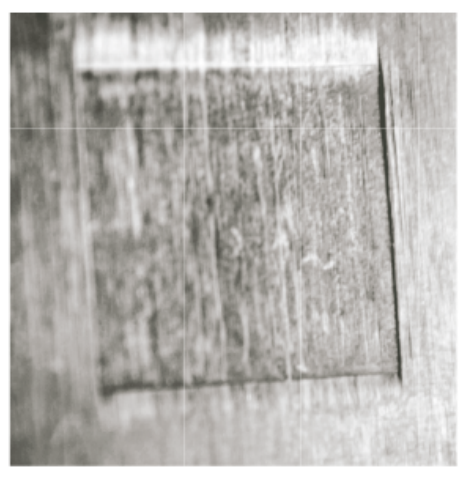

Figure 9. Specimen with delaminations generated by indenting the prepreg before ply layup and cure. The image on the left shows the location and the delaminations and the image on the right shows a typical indentation in the prepreg before ply layup.

Both the as measured response and the noise reduced responses at 3.33 seconds is shown in Fig. 10. The time displayed is relatively long, to allow for the heat to diffuse through the full thickness of the composite and to allow for the possibility of delamination detection between the last two plies in the specimen ( $90 \%$ deep). In the as measured response (left image), the most significant contribution to the contrast is the uneven heating of the specimen. As is the case with the late time response of the impacted composite (Fig. 8), the noise reduction algorithm significantly reduces this effect as is shown in the image on the right. As is evident from an examination of the figure, some of the delaminations that are barely visible in the original data are clearly detectable in the noise reduced image. In particular all of the delaminations at $50 \%$ and $60 \%$ of the total thickness are clearly visible and the three largest delaminations at $70 \%$ of the total thickness are visible. The minimums and maximums from the same region of the images were used to linearly set the gray scale settings of the images.
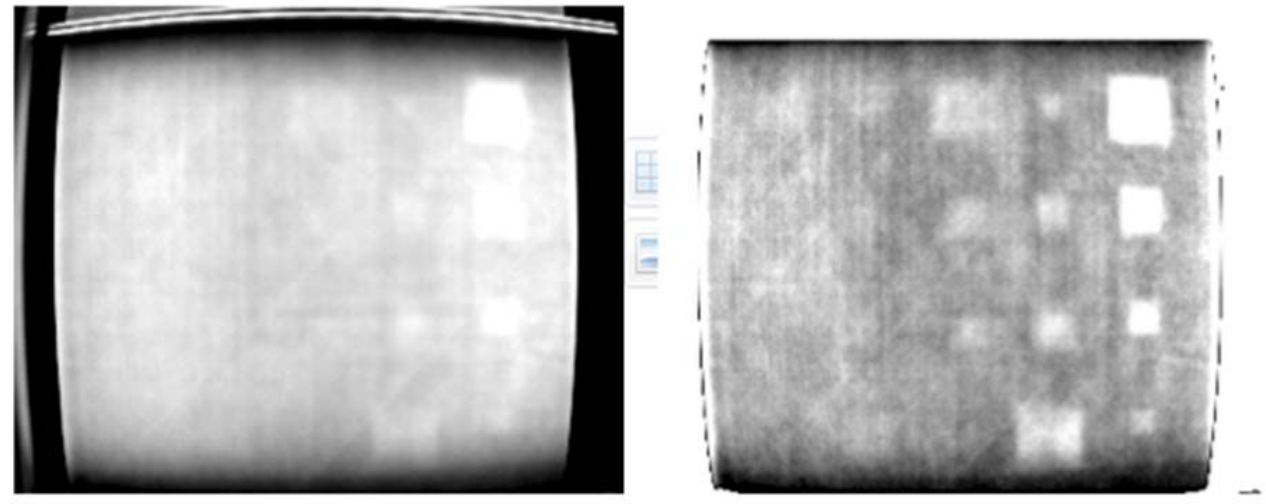

Figure 10. Thermal response of delaminated composite 3.33 seconds after impulse heating as acquired on the left and following the application of the noise reduction technique on the right.

The third composite specimen used to examine the potential of the technique was the same impact composite examined in section 1 for which the thermal response is shown in Fig. 1. This specimen has both impact damage and white paint used to indicate to the technician, where the impact should be performed. In this case, the change in thermal response as a result of the impact is only slightly greater than the "material noise". Additionally, the white paint does not absorb the flash heating as well as the composite surface, therefore there is a cooler region delineating the circle. An examination of the temporal variation of temperature profile across the paint, show that the width of the black circle in the image grows 
with time, indicative of the heat diffusing into the cooler region where the white paint is on the surface. The data as acquire post impact is shown on the far left in Fig. 11. The center image is the same as the right-most image shown in Fig. 1, where the data acquired before the impact is aligned and subtracted from the data acquired post impact. The image on the far right, was obtain using only the data acquired after impact. The effect of thermal variations due to material variations has been significantly reduced, resulting in the variation in the response from the impact being the largest variation in image. The technique is not as good as subtracting the pre impact response, however, it does not require acquiring the pre impact response.
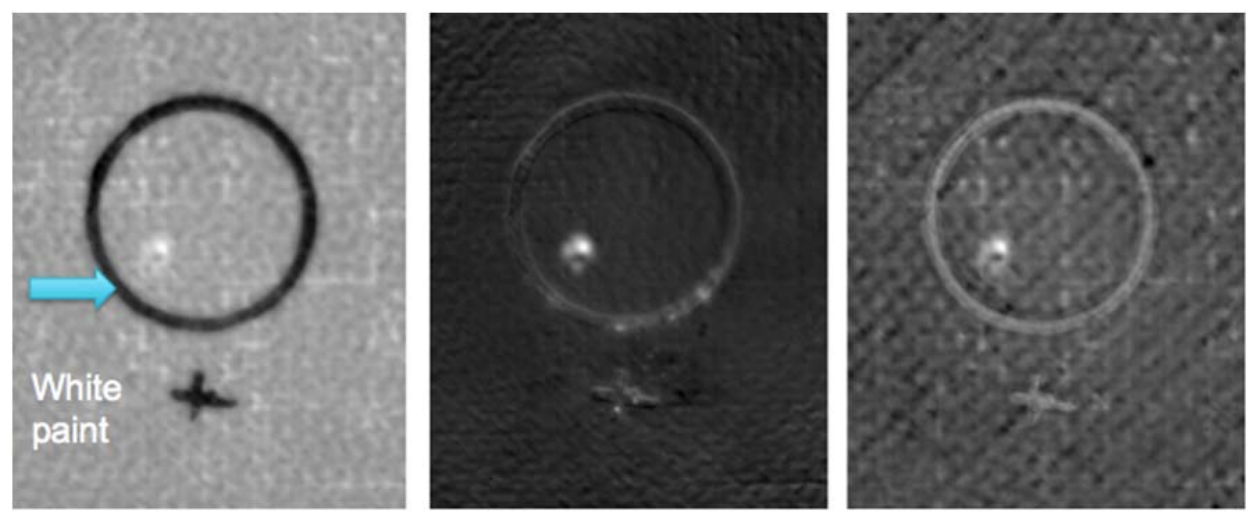

Figure 11. The left and center images are thermographic images acquired on a composite specimen after an impact and the difference between the pre and post impact difference respectively. The image on the right is obtained using the noise reduction algorithm on the post-impact thermal data.

\section{CONCLUSION}

A method has been presented for reducing the effect of near surface thermal variation in a composites thermal response by applying a two dimensional finite difference method to estimate its shape in later times. Application of the method to simulation for a small subsurface void illustrates it is possible to approximately estimate the spatial and temporal response with a two dimensional finite difference equation of just the surface temperature.

The technique was applied to three different composite specimens. While the technique does not perform as well as using data acquired prior to the damage inducing event, it does significantly reduce the material induced noise. For the case of an impacted composite, it reduces the noise by a factor of 5 at early times and a factor of two at later times. An examination of the results of the technique at later times, indicates the method significantly reduces the effect of uneven heating.

\section{REFERENCES}

[1] Bates, D., Smith, G., Lu, D. and Hewitt, J., "Rapid thermal non-destructive testing of aircraft components"

Composites Part B: Engineering, 31, 175-185.(2000)

[2] Almond D., Delpech P., Beheshtey M., Wen P. "Quantitative determination of impact damage and other defects in carbon fibre composites by transient thermography." SPIE Proc. 2944:256-64(1996).

[3] Van Derlofske, J. F., " Thermographie Imaging of Defects in Anisotropie Composites," Review of Progress in Quantitative Nondestructive Evaluation, 457-464(1998)

[4] Avdelidis N.P., Almond D.P., Dobbinson A., Hawtin B.C., Ibarra-Castanedo C., Maldague X., "Aircraft composites assessment by means of transient thermal NDT," Progress in Aerospace Sciences, 40, pp. 143-162(2004).

[5] Kaczmarek, H. and Maison, S. "Comparative ultrasonic analysis of damage in CFRP under static indentation and low-velocity impact," Composites Science and Technology, 51, 11-26(1994) 
[6] Thomas, J.W., [Numerical Partial Differential Equations: Finite Difference Methods],Springer -Verlag New York, Inc, 1995 